\section{Rare-Earth-Doped Bromide Materials Display Lasing Activity at Near-Infrared Transitions}

Long-wavelength infrared light sources are important for a variety of applications, including remote sensing, night vision, and medical diagnosis. However, almost all solid-state materials that exhibit lasing at these wavelengths are easily damaged by moisture, making them difficult to use outside of the laboratory. Now, K. Rademaker, S.A. Payne, and W.F. Krupke of Lawrence

\section{Protein-Based Thermoplastic Elastomers Biochemically Synthesized}

Genetic engineering methods can now be used to prepare multiblock protein copolymers. It is well known that synthetic multiblock copolymers can form biphasic materials that exhibit, to varying degrees, the mechanical and chemical properties of the constituent blocks. Property tuning can be effected by controlling interphase mixing of incompatible blocks. Interphase mixing, in turn, depends on the range of microstructures accessible to the blocks, which is much larger for systems comprising large, well-defined blocks. Recently, researchers at Emory University and the Georgia Institute of Technology demonstrated that, by synthesizing large multiblock protein copolymers, material microstructure at both the nanoscale and mesoscale can be systematically modified in a manner that was previously unfeasible.

As reported in the January 25 issue of Macromolecules (p. 345; DOI: 10.1021/ ma0491199), K. Nagapudi of the Emory University School of Medicine and the Georgia Institute of Technology, E.L. Chaikof of the Emory University School of Medicine, V.P. Conticello of Emory University, and co-researchers used a recombinant DNA technique to synthesize a new class of protein BAB triblock copolymers, whose respective blocks exhibit distinct elastomeric (A) and plastic (B) mechanical properties analogous to synthetic thermoplastic elastomers, where

$$
\begin{aligned}
& \mathbf{A}=\operatorname{VPGVG}\left[(\mathrm{VPGVG})_{2} \mathrm{VPGEG}(\mathrm{VPGVG})_{2}\right]_{48} \mathrm{VPGVG} \text { and } \\
& \mathbf{B}=\text { VPAVG }\left[(\mathrm{IPAVG})_{4}(\mathrm{VPAVG})\right]_{16} \mathrm{IPAVG}
\end{aligned}
$$

Knowing from previous research that elastin-mimetic proteins display an inverse temperature profile for self-assembly, the researchers chose the sequence of the hydrophilic elastomeric A block so that its transition temperature $\left(T_{\mathrm{c}}\right)$ is substantially higher than $37^{\circ} \mathrm{C}$ (physiological temperature) and the sequence of the hydrophobic plastic $\mathbf{B}$ block so that its $T_{\mathrm{C}}$ is close to room temperature. When casting films, the researchers observed that their triblock copolymer protein reversibly self-assembled from concentrated aqueous solution at a temperature above the $\mathbf{B}$ block's $T_{\mathrm{c}}$ to form a network of plastic microdomains dispersed in a continuous elastomeric phase composed of the A blocks. The researchers said that the ability of the $\mathbf{B}$ blocks to form virtual cross-links and to maintain plastic deformation behavior in the absence of chemical or radiation cross-linking was completely unanticipated. Through rational choice of film processing conditions that control mesoscale and nanoscale structure, the researchers observed increases in the Young's modulus of more than three orders of magnitude $(0.03-35 \mathrm{MPa})$ and fivefold increases in elongation to break (250-1300\%).

In order to investigate the effect of processing conditions on drug-release properties, the researchers measured the release rates of a model amphiphilic drug from the protein triblock copolymer. For identical drug-loading conditions, the research team observed a diffusion coefficient in a water-cast film that was 30 times larger than that in a film cast from tetrafluoroethylene.

The researchers said that their novel class of protein polymer illustrates important new design principles for protein-based materials with unique microstructures and properties. In addition, they anticipate that "protein-based thermoplastic elastomers will find applications as novel scaffolds for tissue engineering and as new biomaterials for controlled drug release and cell encapsulation."

STEVEN TROHALAKI
Livermore National Laboratory; E. Heumann and G. Huber of Universität Hamburg, L.I. Isaenko of the Russian Academy of Sciences; and A. Burger of Fisk University have achieved lasing at nearinfrared wavelengths in moisture-resistant, $\mathrm{Nd}^{3+}$-doped $\mathrm{KPb}_{2} \mathrm{Br}_{5}(\mathrm{KPB})$ and $\mathrm{RbPb}_{2} \mathrm{Br}_{5}$ (RPB) crystals. The results, reported in the April 1 issue of Optics Letters (p. 729), suggest that these media may be able to support long-wavelength infrared lasing and be usable in the field.

Rademaker and colleagues grew single $\mathrm{KPB}$ and RPB crystals from a stoichiometric mixture using the Bridgman technique. Since the key limitation for solid-state long-wavelength infrared laser media is nonradiative decay from the lasing levels due to multiphonon interactions, the researchers anticipated that the greater mass of $\mathrm{Br}$ compared to $\mathrm{Cl}$ would reduce the phonon energy relative to previously studied $\mathrm{KPb}_{2} \mathrm{Cl}_{5}$ crystals and allow lasing from previously unusable high-angularmomentum levels. Under optical excitation at $0.75 \mu \mathrm{m}$, the samples displayed strong emission peaks at $1.18 \mu \mathrm{m}, 1.07 \mu \mathrm{m}$, and $0.97 \mu \mathrm{m}$, indicating that the conjecture was correct. The researchers were able to generate laser activity at $1.07 \mu \mathrm{m}$ in flat, uncoated samples of both $\mathrm{Nd}: \mathrm{KPB}$ and $\mathrm{Nd}: \mathrm{RPB}$ and at $1.18 \mu \mathrm{m}$ and $0.97 \mu \mathrm{m}$ in $\mathrm{Nd}: \mathrm{RPB}$, using a $10 \mathrm{~ns}$ pulsed optical parametric oscillator as a pump source.

These results show that moistureresistant, rare-earth-doped alkali lead halide compounds may be the solution for robust long-infrared-wavelength sources. Although Rademaker and colleagues were able to demonstrate lasing only in pulsed operation, they speculate that a higher concentration of the $\mathrm{Nd}$ dopant might enhance cross-relaxation of population from the lower lasing levels and allow for continuous-wave operation. COLIN MCCORMICK

\section{$\mathrm{Si}_{3} \mathrm{~N}_{4}$ Nanobelts Grown by Pyrolysis of Polyureasilazane with Iron Catalyst}

Silicon nitride is known for its robust thermal and mechanical properties as well as its wide-bandgap electronic band structure, in which mid-gap energy levels can be introduced with dopants to tailor its electronic and optical properties. Onedimensional $\mathrm{Si}_{3} \mathrm{~N}_{4}$ nanostructures may make it possible to engineer devices that take advantage of electron confinement effects for use in high-temperature environments. One example of such structures is the $\mathrm{Si}_{3} \mathrm{~N}_{4}$ nanobelt synthesized by $\mathrm{W}$. Yang of Tsinghua University, L. Zhang of the Chinese Academy of Sciences, H. Ji of Peking University, L. An of the University 